\title{
Comparison of the size of eggs from Apis mellifera $L$ queens and laying workers
}

\author{
J Woyke \\ Agricultural University SGGW, Bee Culture Division, \\ 166 Nowoursynowska, 02-766 Warsaw, Poland
}

\begin{abstract}
Summary - The shortest eggs laid by $A m$ mellifera laying workers were shorter, and the longest were longer than eggs deposited by queens. The coefficient of variance for length of eggs deposited by laying workers was 3.5 times higher than for eggs from queens. Eggs from queens were longer $(1.620$ $\mathrm{mm})$, but narrower $(0.368 \mathrm{~mm})$ than eggs from laying workers $(1.579$ and $0.378 \mathrm{~mm})$. Correlation coefficient between length and width was significant for eggs from laying workers but not from queens. Both types of $A$ mellifera eggs were longer than eggs from $A$ florea queens, but shorter than from $A$ florea laying workers.
\end{abstract}

\section{A m mellifera / queen / laying worker / egg/ morphometry}

\section{INTRODUCTION}

The size of thousands of fertilized eggs deposited by Apis mellifera queens during 5 months was measured by Hejtmanek (1961) and Jordan (1961). Henderson (1992) compared size of fertilized and unfertilized eggs deposited by two queens during 2.5 months. According to Borodacheva (1973), queens reared from heavier eggs were heavier and had more ovarioles than those from lighter ones. Weight of eggs affected also the external characters of workers to some extent. Bilash et al (1985) showed that colonies headed by queens reared from heavier eggs produced more honey than those headed by queens hatched from lighter eggs.

Woyke (1975) measured size of eggs from queens of three Asian honey bee species. According to Woyke (1993b), the eggs changed their size during the incubation period. Despite the fact that the length of $A$ florea workers was equal only to the length of queen abdomen the eggs from laying workers were larger than from queens (Woyke, 1993a).

Size of eggs deposited by $A$ mellifera laying workers was till now not measured. Therefore it was interesting to compare the size of eggs laid by queens and laying workers in A mellifera.

\section{MATERIALS AND METHODS}

The investigations were conducted in August 1993. Hybrid A m mellifera bees were investigated. Size of 50 eggs from each colony was measured. Three colonies were made completely 
queenless. When laying workers appeared, their eggs were investigated. At the same time three colonies with queens were selected. A comb with brood of different age was taken from each colony. To assure collection of eggs of different age, they were collected from different places on both sides of a comb. Eggs from colonies with queens and laying workers were carefully collected from comb cells with the aid of a grafting needle. They were put down on a glass microscopic slide and covered with a small drop of vaseline oil to make them transparent. This enabled to measure the size of the embryon in the egg. The size of eggs was measured with the aid of eye-piece micrometer in a stereoscope microscope. Magnification 62.5 times was used to measure the length and 100 times the width of eggs. The length was measured as the distance between the anterior and the posterior poles of the chorion of the egg. The width was measured in the middle of the length of the egg. The length of the body of the embryon was measured neglecting the amnion-seroza (Milne et al, 1988).

Analysis of variance and Newman-Keuls multiple comparison test was applied to determine significant differences among means. The $t$-test was used to compare specific pairs of means. Correlation coefficients between length and width of eggs were calculated.

\section{RESULTS}

\section{Length of eggs}

The length of the embryon in eggs laid by queens ranged from $1.170 \mathrm{~mm}$ to 1.482 $\mathrm{mm}$. According to Woyke (1993a) the embryon changes its length during the incubation period of the egg. Thus, a wide variation indicated that eggs of different age were collected. Results presented in table I show that the length of eggs deposited by three queen bees ranged from 1.560 to $1.690 \mathrm{~mm}$ and by laying workers in three colonies from 1.196 to $1.872 \mathrm{~mm}$. The shortest eggs from laying workers in any colony were shorter and the longest were longer than eggs from any queen. The shortest egg from queen was $30 \%$ longer than the shortest egg from laying worker. The variation in the length of eggs deposited by laying workers was higher than in eggs deposited by queen bees (table 1). The overall coefficient of variance for the length of 150 eggs from three queens was $1.9 \%$ and from laying workers in three bee colonies $6.7 \%$. Thus it was 3.5 times higher for laying workers than for queen bees.

Figure 1 shows that the ranges from 25 to 75 percentiles eggs deposited by queens were much lower than those for laying workers. The span of those ranges (25-75\%) for eggs from laying workers were almost equal to that of the total range ( $\min$ - max) of eggs from queens. The median value for the length of eggs deposited by each of the three queens was $1.61 \mathrm{~mm}$, by laying workers in two colonies $1.59 \mathrm{~mm}$ and in the third colony $1.56 \mathrm{~mm}$. Thus all were lower in laying workers than in queens.

The mean length of eggs laid by queens ranged from 1.612 to $1.624 \mathrm{~mm}$ and deposited by laying workers from 1.550 to $1.597 \mathrm{~mm}$. No statistically significant differences were found between means of eggs from the queens (table 1). The absolute means of eggs from laying workers were lower than from queen bees. However, only mean length of eggs from laying workers in colony no 6 was significantly lower than any mean length of eggs from queens or laying workers in the two other colonies. The overall mean for the length of eggs from all queens was $1.620 \mathrm{~mm}$ ( $\mathrm{SD}=0.031, n=$ $150)$ and from laying workers in all three colonies was $1.579 \mathrm{~mm}$ (SD $=0.105, n=$ $150)$. The $t$-test $(t=4.48, \mathrm{df}=298, P=<$ 0.001 ) showed that eggs from queens were highly significantly longer than eggs from laying workers.

\section{Width of eggs}

The width of eggs laid by all three queens ranged from 0.350 to $0.403 \mathrm{~mm}$ and 
deposited by laying workers in three colonies from 0.263 to $0.560 \mathrm{~mm}$ (table I). The minimal as well as the maximal values were identical for all queens. They differed for eggs from laying workers. The narrowest eggs laid in any colony by laying workers were narrower and the widest were wider than any egg laid by any queen. The narrowest eggs from a queen was $33 \%$ wider than from the laying worker and the widest from the laying worker was $39 \%$ wider than from a queen. The variation in the width of eggs from laying workers in any colony was higher than from any queen (table I). The overall coefficient of variance for 150 eggs laid by three queens was $4.0 \%$ and for eggs deposited by laying workers $11.6 \%$. Thus, the coefficient was three times higher for eggs from laying workers than from queen bees. Figure 2 shows that the span of range from 25 to 75 percentiles was for laying worker eggs in two colonies larger than for eggs laid by queen bees. The median values for width of eggs from all three queens and laying workers in one colony were equal $(0.37 \mathrm{~mm})$. The medians for laying worker eggs in two other colonies were $0.38 \mathrm{~mm}$.

The mean width of eggs deposited by three queens $(0.367-0.369)$ was very similar and did not differ significantly. Eggs from laying workers $(0.359-0.393)$ were in two colonies significantly wider than in the third one. The overall mean of the width of eggs deposited by queens was $0.368 \mathrm{~mm}$ (SD = $0.014, n=150$ ) and by laying workers 0.378 $\mathrm{mm}(\mathrm{SD}=0.438, n=150)$. The $t$-test $(t=$ 2.54 , df $=298, P=0.006$ ) showed that on the average eggs from laying workers were significantly wider than from queen bees.

\section{Relationship between length and width of eggs}

The correlation coefficient for the six pairs of means for length and width $r=0.149$ ( $d f=4$,
$P=0.779$ ) did not indicate significant correlation.

Correlation coefficients for three individual queens $r=0.272, P=0.056, r=0.080$, $P=0.579$ and $r=-0.042, P=0.775$ also did not indicate significant correlation between length and width of eggs. Those coefficients for eggs of laying workers in three colonies $r=-0.035, P=0.811 ; r=$ $0.372, P=0.013$ and $r=0.442, P=0.001$ proved significant or highly significant correlations for two colonies. Correlation coefficients for length and width of eggs deposited by one queen and laying workers in one colony were negative, indicating compensation for those characters. Thus longer eggs from these colonies were narrower. The overall correlation coefficient for all 150 pairs of measurements from three queens $r=0.536$, df $=148, P=0.514 \mathrm{did}$ not indicate significant correlation. However, the coefficient for laying workers eggs from all three colonies $r=0.249$, df $=148, P=$ 0.02 proved low but significant correlation. Thus longer eggs were also wider.

\section{DISCUSSION}

All three authors (Hejtmanek, 1961; Jordan, 1961; Henderson, 1992) presented variation in eggs size throughout the season. Egg sizes found in this paper were similar to those presented by Hejtmanek (1961), a little larger than those (probably of $A \mathrm{~m}$ carnica) by Jordan (1961), and a little smaller than those (probably of $A$ m ligustica) by Henderson (1992). Thus size of eggs deposited by queens of different races apparently differs. Henderson (1922) did not find differences in size of fertilized and unfertilized eggs deposited by queens. In this paper significant differences between size of eggs from queens and laying workers were found. The most striking was the three times higher variation in eggs from laying workers than from queens. It can be 
expected that the size of eggs from laying workers will also vary throughout the season. However, it can be assumed that the most striking difference will remain. Although eggs change their size during the incubation period (Woyke, 1993b) it can be supposed that in this investigation eggs of varying age were collected from each colony.

A $m$ mellifera eggs were smaller than eggs of three Asian honey bee species (Woyke, 1975). Eggs of A florea from India were larger than from Thailand (Woyke, 1993a). This could be caused by different race as well as by different environmental conditions.

Comparing the size of eggs deposited by queens (q) and laying workers (w) of $A m$ mellifera (Am) and $A$ florea (Af) from Thailand, the following sequence was found (in $\mathrm{mm}$ ) for length: $1.572 \mathrm{Afq}, 1.579 \mathrm{Amw}$, $1.620 \mathrm{Amq}, 1.703 \mathrm{Afw}$; and width: 0.368 Amq, $0.371 \mathrm{Afq}, 0.378 \mathrm{Amw}, 0.436 \mathrm{Afw}$. Thus, contrary to $A$ florea, eggs from $A m$ mellifera queens were longer than from laying workers. However, A florea eggs from queens were shorter and from laying workers longer than both types of $A m$ mellifera eggs. Similarly like in $A$ florea, eggs from $A$ mellifera laying workers were wider than from queens. However, eggs from A florea laying workers were still wider. Thus the narrowest were eggs from $A m$ mellifera queens and the widest from $A$ florea laying workers. Hence, eggs from $A$ florea laying workers were longer and wider than any type of A $m$ mellifera eggs.

Depending upon the season and race of honeybee compared, the relationship between the size of eggs from different subspecies may differ. However, investigations conducted till now showed that eggs from queens and laying workers differed in $A$ florea (Woyke, 1992a) as well as in $A m$ mellifera. It can be supposed that such differences occur also in the other subspecies and races of honey bee.

Although significant correlations were found for length and width of $A$ florea eggs from queens and laying workers, in $A \mathrm{~m} \mathrm{mel-}$ lifera a significant correlation was found only for eggs from laying workers.

\title{
Deutsche Version
}

\section{Größenvergleich von Eiern der Königinnen und legenden Arbeiterinnen bei Apis mellifera $\mathrm{L}$}

\begin{abstract}
Zusammenfassung - Die kürzesten Eier der Arbeiterinnen waren kürzer und die längsten waren länger als Eier der Königinnen. Der Varianzkoeffizient der Eilänge ist bei Arbeiterinneneiern 3,5 mal höher als für Eier der Königinnen. Im Durchschnitt waren die Eier der Königinnen länger $(I=1,620 \pm$ $0,03 \mathrm{~mm})$ aber dünner $(\mathrm{d}=0,368 \pm 0,014 \mathrm{~mm})$ als die Eier der Arbeiterinnen $(I=1,579 \pm 0,105 \mathrm{~mm}$ und $d=0,378 \pm 0,438 \mathrm{~mm}$ ). Der Korrelationskoeffizient zwischen Länge und Durchmesser war für die Arbeiterinneneier signifikant, nicht aber für Königinneneier. Die Eier beider $A$ mellifera waren länger als die $\operatorname{der} A$ florea Königinnen aber kürzer als die der $A$ florea Arbeiterinnen.
\end{abstract}

A m mellifera / Königin / legende Arbeiterinnen / Eier / Morphometrie 


\section{EINLEITUNG}

Die Größe von Tausenden befruchteter Eier von $A$ mellifera Königinnen wurde über eine Zeit von 5 Monaten von Hejtmanek (1961) und Jordan (1961) gemessen. Henderson (1992) fand keinen Größenunterschied zwischen befruchteten und unbefruchteten Eiern von 2 Königinnen während 2,5 Monaten. Königinnen, die aus schweren Eiern gezogen wurden waren schwerer und hatten mehr Ovariolen als solche von leichten Eiern. Das Eigewicht hatte auch einen gewissen Einfluß auf die äußerlichen Eigenschaften der Arbeiterinnen (Borodacheva, 1973). Nach Bilash et al (1985) produzieren Völker mit Königinnen, die aus schweren Eiern stammen mehr Honig als solche, deren Königinnen aus leichteren Eiern stammen.

Woyke (1975) bestimmte die Eigröße der Königinnen von 3 asiatischen Honigbienenarten. Dabei zeigte sich, daß die Eier ihre Form während der Entwicklung änderten. Obwohl eine A florea Arbeiterin viel kleiner als eine Königin ist (sie ist insgesamt nicht größer als der Hinterleib einer Königin) waren ihre Eier größer als die der Königin (Woyke, 1993a). Bisher ist die Größe von Arbeiterinneneiern bei $A$ mellifera unbekannt. So ist es wichtig, den Größenvergleich von Königinnen- und Arbeiterinneneiern durchzuführen.

\section{MATERIAL UND METHODE}

Die Messungen wurden im August 1993 mit Hybriden der $A$ m mellifera durchgeführt. Dabei wurden je 50 Eier von 3 Königinnen und 3 Völkern mit eierlegenden Arbeiterinnen gemessen. Dazu wurde jeweils eine Wabe mit Brut verschiedenen Alters genommen, und die Eier wurden von verschiedenen Stellen von beiden Seiten mit einem Umlarvlöffel entnommen, um möglichst alle Altersstufen in den Proben zu haben. Die Eier wurden auf einen Objektträger gelegt und mit Vaselinöl beträufelt, damit sie durchsichtig wurden. Dadurch konnte man die Größe des Embryos bestimmen. Die Eilänge wurde mit einem Meßokular bei einer Vergrößerung von 62,5, der Durchmesser bei einer 100-fachen Vergrößerung im Stereomikroskop gemessen. Als Länge wurde der Abstand zwischen den vorderen und hinteren Polen des Chorions genommen, der Durchmesser wurde in der Mitte des Eies gemessen. Die Länge des Embryos wurde ohne die Amnionhülle bestimmt (Milne et al, 1988).

\section{ERGEBNISSE}

\section{Die Eilänge}

Die Länge des Embryos in Ejern von Königinnen variierte von 1,170 bis $1,482 \mathrm{~mm}$. Diese große Variation zeigt, daß Eier verschiedener Altersstufen gemessen wurden. Die Eilänge von 3 Königinnen reichte von 1,560 bis $1,690 \mathrm{~mm}$, die der Arbeiterinnen von 1,196 bis $1,872 \mathrm{~mm}$. Demnach ist die Länge der Arbeiterinneneier wesentlich variabler als die der Königinneneier (Tabelle I). Das kürzeste Arbeiterinnenei ist $30 \%$ kürzer als das kleinste Ei einer Königin. Der Varianzkoeffizient für die Eilänge von 150 Eiern von 3 Königinnen war 1,9\%, der entsprechende für Arbeiterinnen war 6,7\%. Nach Überführung der Rohwerte in Perzentile zeigt der Bereich von 25 bis 75 bei Königinneneiern wesentlich kleinere Längenunterschiede als bei Arbeiterinnen (Abb 1). Der gesamte Schwankungsbereich der Eilängen bei den Königinnen ist fast genauso groß wie der Bereich von 25-75 Perzentilen bei den Arbeiterinnen.

Der Median der Eilänge beträgt bei allen 3 Königinnen $1,610 \mathrm{~mm}$, bei den Arbeiterinnen von 2 Völkern 1,59 und vom 3. Volk $1,56 \mathrm{~mm}$. Die durchschnittliche Eilänge für jede Königin lag zwischen 1,612 und 1,624 $\mathrm{mm}$, die der einzelnen Völker mit eierlegenden Arbeiterinnen zwischen 1,550 und $1,597 \mathrm{~mm}$. Diese Unterschiede sind nicht signifikant, mit einer Ausnahme: Die 
Eigrößen der Arbeiterinnen des Volkes 6 sind signifikant kleiner als alle anderen Eier. Faßt man dagegen die Werte der 3 Königinnen zusammen $(1,620 \mathrm{~mm} \pm 0,031, n=$ 150) und vergleicht sie mit dem Gesamtmittelwert der Arbeiterinnen der 3 Völker $(1,579 \pm 0,105, n=150)$ so ergibt sich ein signifikanter Unterschied ( $t$-Test, $t=4,48$, df $=298, P<0,001$ ).

\section{Durchmesser der Eier}

Der Durchmesser der Königinneneier schwankte zwischen 0,350 und $0,403 \mathrm{~mm}$, die der Arbeiterinneneier zwischen 0,263 und 0,560 (Tabelle I). Bei den Durchmessern zeigte sich wie bei den Eilängen, daß die Schwankungsbreite bei den Eiern der Arbeiterinnen wesentlich höher ist als bei den Königinnen. Das dünnste Königinnenei war $33 \%$ dicker als das dünnste Arbeiterinnenei, das dickste Königinnenei war 39\% dünner als das dickste Arbeiterinnen-ei. Der Varianzkoeffizient von 4,0\% für Königinneneier war fast um $1 / 3$ kleiner als der der Arbeiterinneneier $(11,6 \%)$. Berücksichtigt man nur die Perzentile von 25 bis 75 (Abb 2), so sind die Größenunterschiede bei 2 Völkern mit legenden Arbeiterinnen wesentlich größer als bei den Königinnen. Der Median der Durchmesser beträgt bei 3 Königinnen und einem Volk mit eierlegenden Arbeiterinnen $0,37 \mathrm{~mm}$. Bei den beiden anderen Völkern beträgt er $0,38 \mathrm{~mm}$.

Die Mittelwerte für die Eier der Königinnen $(0,367-0,369)$ unterscheiden sich nicht signifikant. Die Eier der Arbeiterinnen von 2 Völkern sind signifikant dicker als bei dem 3.Volk. Die Gesamtmittelwerte aller 3 Königinnen $(0,368 \pm 0,014, n=150)$ unterscheiden sich signifikant von denen aller Arbeiterinnen $(0,378 \pm 0,438)$ im $t$-Test $(t=$ $2,54, \mathrm{df}=298, P=0,006)$.

\section{Verhältnis Länge zu Durchmesser}

Der Korrelationskoeffizient aus mittleren Längen und Durchmessern der Eier zeigte weder für die 6 Paare noch für die 3 Königinnen eine Signifikanz. Bei den Arbeiterinneneiern ergab sich jedoch bei 2 Völkern eine signifikante Korrelation $(r=0,372, P=$ 0,013 und $r=0,442, P=0,001$ ). Bei einem Volk zeigte sich ein negativer Korrelationskoeffizient $(r=-0,035)$, ein ähnlicher negativer Wert bestand auch bei einer Königin $(r=-0,042)$. In diesen Fällen kompensierten sich die beiden Eigenschaften, dh längere Eier sind dünner. Der Koeffizient für alle 150 Arbeiterinneneier zeigte allerdings eine geringe Korrelation $(r=0,249, \mathrm{df}=148, P=$ $0,02)$, so daß die längeren Eier auch einen größeren Durchmesser haben, also insgesamt größer sind.

\section{DISKUSSION}

Die 3 Autoren Hejtmanek (1961), Jordan (1961) und Henderson (1992) wiesen eine Variation der Eigröße in Abhängigkeit von der Jahreszeit nach. Die Eigrößen, die jetzt für $A m$ mellifera bestimmt werden, sind ähnlich wie die von Hejtmanek, etwas größer als die von Jordan (wahrscheinlich $A$ $m$ carnica) und etwas kleiner als die von Henderson (wahrscheinlich $A$ m ligustica). Demnach unterscheiden sich die Eigrößen bei den Rassen.

In dieser Untersuchung wurden signifikante Größenunterschiede zwischen Eiern von Königinnen und Arbeiterinnen nachgewiesen. Da zwischen befruchteten und unbefruchteten Eiern von Königinnen kein Größenunterschied besteht (Henderson, 1992), war es nicht nötig, Drohneneier der Königinnen zu berücksichtigen. Das erstaunlichste Ergebnis ist die 3 mal höhere Varia- 
tion in der Größe bei den Arbeiterinneneiern im Vergleich zu den Eiern der Königinnen. Es ist anzunehmen, daß auch bei den Arbeiterinneneiern eine jahreszeitliche Größenabhängigkeit besteht. Obwohl die Eier ihre Form mit der Entwicklung des Embryos ändern (Woyke, 1993b), haben diese Unterschiede wegen der Berücksichtigung der Eier verschiedener Altersstufen bei der hier vorgelegten Untersuchung keinen Einfluß auf die Ergebnisse.

A $m$ mellifera Eier waren kleiner als die Eier der 3 asiatischen Honigbienenarten (Woyke, 1975). Eier der indischen A florea waren größer als die von Thailand (Woyke, 1993a). Dieser Unterschied könnte auf verschiedene Rassen oder auf Umwelteinflüsse zurückzuführen sein.

Vergleicht man die Eier von Königinnen (K) und Arbeiterinnen (A) bei A m mellifera (Am) und $A$ florea (Af) von Thailand, läßt sich folgende Reihe aufstellen: Länge 1,572 $\mathrm{mm}$ AfK, $1,579 \mathrm{~mm}$ AmA, 1,620 mm AmK und 1,703 mm AfA; Durchmesser: 0,368 $\mathrm{mm}$ AmK, 0,371 mm AfK, 0,378 mm AmA und $0,436 \mathrm{~mm}$ AfA.

Somit sind die Königinneneier bei $A$ mellifera länger als die Arbeiterinneneier. Das ist bei $A$ florea anders. Hier legen die Königinnen die kürzeren, ihre Arbeiterinnen die längeren Eier. Im Vergleich zu A mellifera haben $A$ florea Königinnen die kürzesten, A florea Arbeiterinnen die längsten Eier. Insgesamt stammten die größten Eier von $A$ florea Arbeiterinnen. Ähnlich wie bei $A$ florea hatten die $A$ mellifera Arbeiterinneneier einen größeren Durchmesser als die Königinneneier. Die signifikante Korrelation zwischen Eilänge und Durchmesser, die sich bei $A$ florea bei beiden Eitypen zeigte, war bei $A$ mellifera nur bei Arbeiterinneneiern nachzuweisen.
Die Eigrößen mögen sich in Abhängigkeit von der Saison und der Rasse ändern. Die Untersuchungen jedoch, die bis jetzt durchgeführt wurden, zeigen, daß bei beiden Bienenarten $A$ florea und $A$ mellifera Unterschiede in der Größe zwischen Arbeiterinnen- und Königinneneiern bestehen. Es ist anzunehmen, daß auch bei den anderen Arten Unterschiede bestehen.

(Übersetzung: N Koeniger)

Résumé - Étude comparative de la taille
des œufs chez les reines d'Apis mellifera et les ouvrières pondeuses. La longueur et la largeur de 50 œufs provenant de 3 colonies avec reines et de 3 colonies avec ouvrières pondeuses (OP) ont été mesurées. Les œufs les plus courts et les œufs les plus longs pondus par les OP étaient respectivement plus courts et plus longs que ceux pondus par les reines (tableau I). L'œuf le plus court d'une reine était $30 \%$ plus long que le plus court d'une OP. Le coefficient de variance pour la longueur des œufs des OP était 3,5 fois plus élevé que celui des œufs des reines (fig 1). La moyenne globale de la longueur et de la largeur des œufs pondus par les reines était respectivement de $1,62 \mathrm{~mm}$ et de 0,368 $\mathrm{mm}$ et de $1,579 \mathrm{~mm}$ et $0,378 \mathrm{~mm}$ pour ceux pondus par les OP. Les œufs des reines sont donc plus longs et plus étroits que ceux des OP. Le coefficient de corrélation entre la longueur et la largeur était significatif pour les œufs des ouvrières pondeuses mais pas pour ceux des reines. Les œufs d' $A \mathrm{~m}$ mellifera, que ce soit de reine ou d'OP, sont plus longs que ceux des reines d'A florea, mais plus courts que ceux de ses ouvrières pondeuses.

\section{A $m$ mellifera / reine / ouvrière pondeuse / œuf / morphométrie}




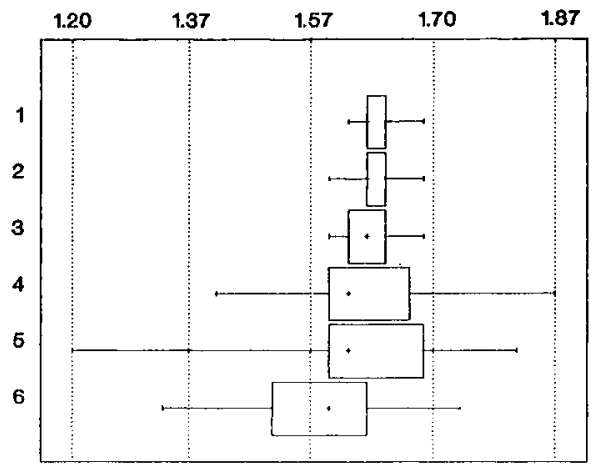

Fig 1. Distribution of $0,25,50,75$ and 100 percentile of length of $A$ mellifera eggs from queens (No 1-3) and laying workers (No 4-6). Numbers on top present length in $\mathrm{mm}$.

Abb I. Verteilung von 0, 25, 50, 75 and $100 \mathrm{Per}$ zentilen der Eilänge von Königinnen ( $\mathrm{Nr} 1-3)$ und legenden Arbeiterinnen ( $\mathrm{Nr}$ 4-6). Die oberen Zahlen geben die Eilänge in $\mathrm{mm}$ an.

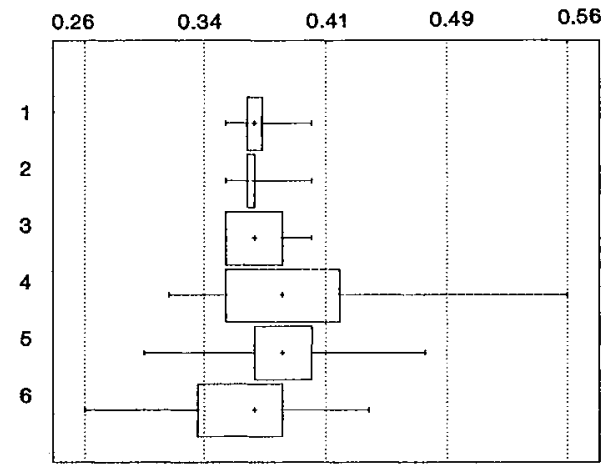

Fig 2. Distribution of $0,25,50,75$ and 100 percentile of width of $A$ mellifera eggs from queens (No 1-3) and laying workers (No 4-6). Numbers on top present width in $\mathrm{mm}$.

Abb 2. Verteilung von 0, 25, 50, 75 und 100 Perzentilen des Durchmessers von Eiern von Königinnen ( $\mathrm{Nr}-3$ ) und legenden Arbeiterinnen $(\mathrm{Nr}$ 4-6). Die oberen Zahlen geben den Durchmesser in $\mathrm{mm}$ an.

Table I. Size (mm) of Apis mellifera eggs deposited by queens and laying workers. Fifty eggs were measured from each colony.

Tabelle I. Eigröße ( $\mathrm{mm}$ ) von Königinnen ( $\mathrm{Nr} 1-3)$ und legenden Arbeiterinnen ( $\mathrm{Nr} 4-6)$ bei A mellifera Von jedem Volk wurden 50 Eier gemessen.

Females

Length

1. Queen

2. Queen

3. Queen

4. Laying workers

5. Laying workers

6. Laying workers

Width

1. Queen

2. Queen

3. Queen

4. Laying workers

5. Laying workers

6. Laying workers
Range

$1.586-1.690$

$1.560-1.690$

$1.560-1.690$

$1.403-1.872$

$1.196-1.820$

$1.326-1.742$
$M e a n \pm S D$

$1.624 \pm 0.0316 \mathrm{~b}$

$1.623 \pm 0.0320 \mathrm{~b}$

$1.612 \pm 0.0273 b$

$1.591 \pm 0.0927 \mathrm{~b}$

$1.597 \pm 0.1197 \mathrm{~b}$

$1.550 \pm 0.0973 a$

$0.369 \pm 0.0142 a b$

$0.367 \pm 0.0125 \mathrm{ab}$

$0.368 \pm 0.0165 \mathrm{ab}$

$0.393 \pm 0.0535 \mathrm{c}$

$0.384 \pm 0.0307 \mathrm{bc}$

$0.359 \pm 0.0374 a$
V\%

\section{9}

2.0

1.7

5.8

7.5

6.3

3.9

3.4

4.5

13.7

8.0

10.4

1 Different letters indicate significant differences among means $(P<0.05)$.

1 Unterschiedliche Buchstaben bedeuten signifikante Unterschiede. 


\section{REFERENCES}

Bilash GD, Borodacheva VT, Timosinova AE (1985) Quality of artificially reased queen bees. Proc XXIXth Int Congr Apicult. Budapest 1983. Apimondia Publ House, Bucharest, 114-118

Borodacheva VT (1973) Weight of eggs and quality of queens and bees. Pchelovodstvo 93 (9), 12-13 (in Russian)

Hejtmanek J (1961) The variability of honeybee eggs. Vedecke Prace Vyzkum Ustav Vcelar, CSAZV 1960, 2, 169-196 (in Czech, Eng summary)

Henderson CE (1992) Variability in the size of emerging drones and of drone and worker eggs in honey bee (Apis mellifera $\mathrm{L}$ ) colonies. J Apic Res 31, 114-118
Jordan R (1961) Über die Beziehung der Eigrösse zum Umfang der Eiablage. Bienenvater 82, 132-134

Miłne CP, Phillips JP, Krell PJ (1988) A photomicrographic study of worker honeybee embryogenesis. J Apic Res 27, 69-83

Woyke J (1975) Eggs in comb cells of three species of Indian bees. Proc 25th int Beekeeping Congr. Grenoble, 321-322

Woyke J (1993a) Occurence and size of laying workers eggs in A florea colonies. J Apic Res $31(3 / 4), 124-127$

Woyke J (1993b) Size change of eggs during the incubation period in three Asian honeybee species. In: Proc 1st Symp Asian Honey Bees and Mites. Bangkok, 197-205 